\title{
Severity of negative symptoms significantly affects cognitive functioning in patients with chronic schizophrenia: The slowing in cognitive processing
}

\author{
Flavia S. Galaverna* \\ Carlos A. Morra** \\ Adrián M. Bueno* \\ * Laboratory of Experimental Psychology, \\ Faculty of Psychology, National University \\ of Córdoba, Córdoba \\ ** Prof. León S. Morra Sanitarium, Córdoba \\ ARGENTINA
}

\begin{abstract}
Background and Objectives: Studies in patients with schizophrenia have shown a decreased overall cognitive performance, and it was found that processing speed and working memory functions are affected. The aim of this study was to describe the general cognitive performance of patients with chronic schizophrenia and analyze its relationship with the severity of psychotic symptoms.

Methods: Forty-eight patients diagnosed with DSM IV-TR schizophrenia disorder were examined for symptom improvement, measured by scales SAPS and SANS. Participants also completed the full scale WAIS-III.

Results: The results show a generalized cognitive deficit, reflected in the low level of general intelligence, as well as the different index that comprise the scale. The most compromised index was the processing speed. The correlations showed that the overall severity of negative symptoms significantly affects cognitive functioning of chronic patients. The formal thought disorder and alogia significantly correlated with almost all the WAIS-III measures.

Conclusions: Multiple studies of specific cognitive domains in schizophrenia have shown that deficits in processing speed are the core element of cognitive impairment in schizophrenia. We support the hypothesis about the slowing in cognitive processing affect both the performance of the basic and more complex cognitive task.
\end{abstract}

Received: 20 November 2013

Revised: 21 July 2014

Accepted: 24 July 2014 


\section{Introduction}

The cognitive profile of individuals with schizophrenia includes alterations in attention functions, memory, language, executive functions and general intelligence ${ }^{2,3}$. According to some authors, the changes are not global and widespread, but specific and targeted, expressed by different patterns of association and dissociation in the performance of different cognitive tasks ${ }^{4}$. Schizophrenia and schizoaffective disorder share a similar pattern of cognitive impairment that is different from that observed in major depression, bipolar disorder and Alzheimer's dementia 5 . There is an increasing interest in the field of research in psychopathology to recognize the importance of these cognitive deficits, in order to understand the etiology, course and outcome of the illness ${ }^{6}$.

Different neuropsychological instruments and batteries have been used to assess these deficits; the Adult Intelligence Scale WAIS$\mathrm{III}^{7}$, has been one of them. A shortened version of it has been used to predict the IQ of these patients ${ }^{8}$, which is believed to be more convenient as the full scale test involves too much time, especially for this type of clinical population. In a study of 42 participants diagnosed with schizophrenia, the average IQ scores were in the low average range indicating a decrease in performance compared to the general population 7 . More specifically, the mean scores of patients in the Verbal Comprehension Index and Perceptual Organization indexes were higher than the Working Memory and Processing Speed. These results indicate the deteriorated state of tasks involving attention, processing speed and working memory ${ }^{7}$. Another study reported that verbal skills in outpatients with schizophrenia assessed by the WAIS are related to the magnitude of the decline in neurocognitive function and performance in everyday life, showing that the processing speed differentiated patients with decreased verbal skills ${ }^{9}$. This could lead to the hypothesis of a close association between verbal performance and the speed in cognitive processing widely reported in the literature ${ }^{10,11}$.

The present study aims to assess and describe the cognitive functioning of a group of inpatients diagnosed with chronic schizophrenia using WAIS-III ${ }^{7}$. This will allow a comparative analysis between different cognitive tasks, some dominated by executive components and others with predominant verbal components. At the same time, we also used different indexes of the scale to investigate specific cognitive domains such as verbal comprehension, perceptual organization, working memory and processing speed. These different measures were also analyzed regarding psychiatric symptoms. There is evidence on the relationship between positive and negative symptoms with the performance on neuropsychological tests. However, there is no model that can associate the presence of specific cognitive impairments and certain symptoms. Additionally there are no studies so far on the relationship between symptoms and cognitive deficits throughout the course of time. It has been established that there is a strong association between these alterations and negative symptoms in a special way. However, this could arise from a conceptual overlap, wherein certain symptoms or cognitive problems are considered as well as negative symptoms and cognitive deficits ${ }^{12}$.

\section{Method}

\section{Subjects}

The study included 48 patients diagnosed with chronic schizophrenia of the mental health institutions of "Prof. León S. Morra" 
Sanitarium and the "San Nicolás" Clinic in the city of Córdoba, Argentina. Patients fulfilled the criteria for Schizophrenia Disorder DSM IV-TR ${ }^{13}$ based on the Structural Clinical Interview for DSM-IV. Patients are between 27 and 60 years of age, of both sexes and different educational levels, and most have not completed their studies. Table 1 shows a detail of the demographic traits. The patients had to be psychiatrically stable for at least two weeks on their treatment with an- tipsychotic drugs before participating in the study. All patients were receiving antipsychotic medication (typical $[n=10]$, atypical $[n=13]$, typical and atypical $[n=25])$. Antipsychotic administration most common were: Clorpromazine, Haloperidol, Levopromazine, Risperidone and Clozapine. All participants were clearly informed regarding the aim of the study and assessment manipulations, and gave their written informed consent prior the beginning of the study.

Table 1

Demographic and clinical characteristics of patients.

\begin{tabular}{|c|c|c|}
\hline Variable & Mean & $\mathrm{SD}$ \\
\hline Age (years) & 48.47 & 12.53 \\
\hline Gender (male / female) & $18 / 25$ & \\
\hline Handedness (right / left) & $42 / 1$ & \\
\hline Education (years) & 10.95 & 3.98 \\
\hline \multicolumn{3}{|l|}{ Subtype } \\
\hline Paranoid / disorganized / residual & $20 / 10 / 13$ & \\
\hline Years of illness & 25.28 & 13.38 \\
\hline SAPS $^{a}$ & 50.73 & 35.89 \\
\hline $\mathrm{SANS}^{\mathrm{b}}$ & 53.32 & 30.24 \\
\hline Hallucination & Moderate & - \\
\hline Delusions & Moderate & - \\
\hline Bizarre behavior & Mild & - \\
\hline Formal thought disorder & Mild & - \\
\hline Affective flattening or blunting & Moderate & - \\
\hline Alogia & Mild & - \\
\hline Avolition / apathy & Mild & - \\
\hline Anhedonia / asociality & Moderate & - \\
\hline Attention & Mild & - \\
\hline
\end{tabular}

a Scale for the Assessment of Positive Symptoms.

${ }^{\mathrm{b}}$ Scale for the Assessment of Negative Symptoms. 


\section{Measures}

\section{Symptom assessment}

Patients were assessed with the Spanish version (available on demand from Dr. Nancy Andreasen) for the Scale for the Assessment of Negative Symptoms -SANS- ${ }^{14}$ and the Spanish version (available on demand from Dr. Nancy Andreasen) for the Scale for the Assessment of Positive Symptoms -SAPS_- ${ }^{15}$.

\section{Cognitive assessment}

The spanish version of Wechsler Adult Intelligence Scale- Third Edition WAIS-III ${ }^{7}$ was a test used to measure intelligence in patients with chronic schizophrenia. The following measures of WAIS-III were considered for this study: Full Scale IQ, Verbal IQ, Performance IQ, Verbal Comprehension, Perceptual Organization, Working Memory and Processing Speed.

\section{Data analysis}

The descriptive statistics mean and standard deviation were calculated to analyze the performance of patients in the different WAIS-III indexes. Pairwise post hoc comparisons were performed with Bonferroni's adjustment. The Pearson correlation coefficient was used to analyze the relationship between cognitive performance and psychotic symptoms. $Z$-scores were calculated to analyze correlations between cognitive performance of patients and the severity of psychotic symptoms. Results were considered significant at $p<0.05$. All statistical tests were carried out using PAWS statistics 18 .

\section{Results}

The results show that in general patients with chronic schizophrenia had a significantly lower performance than expected (Figure 1). Descriptive results showed a low performance in the intelligence test, with an average IQ of 70 points which is the inferred borderline intellectual functioning. The results of the verbal scale were about 10 points higher that the non-verbal performance scales, qualitatively assessed as extremely low (Table 2).

Table 2

Descriptive results for the performance in the indexes WAIS-III in patients with chronic schizophrenia.

\begin{tabular}{lcc} 
IQ Scores / Index Scores & Mean & SD \\
\hline IQ Full Scale & 70.20 & 13.38 \\
Verbal IQ & 76.38 & 14.75 \\
Performance IQ & 67.67 & 10.40 \\
Verbal Comprehension & 81.20 & 18.15 \\
Perceptual Organization & 71.73 & 11.29 \\
Working Memory & 68.47 & 9.51 \\
Processing Speed & 66.53 & 7.86 \\
\hline
\end{tabular}

By analyzing the different indexes that compose the scale WAIS-III, a significant effect between them was observed $(F=$ $15.201 ; p=0.000$ ). Pairwise post hoc comparisons were performed with Bonferroni's adjustment. As shown in Table 3, there are significant differences for each paired comparison for all of the indexes scores except for indexes working memory and processing speed. More specifically, the verbal comprehension index was higher compared to the other index, but still lower than expected and on average low. The processing speed index was the lowest, falling into the category of extremely low. 


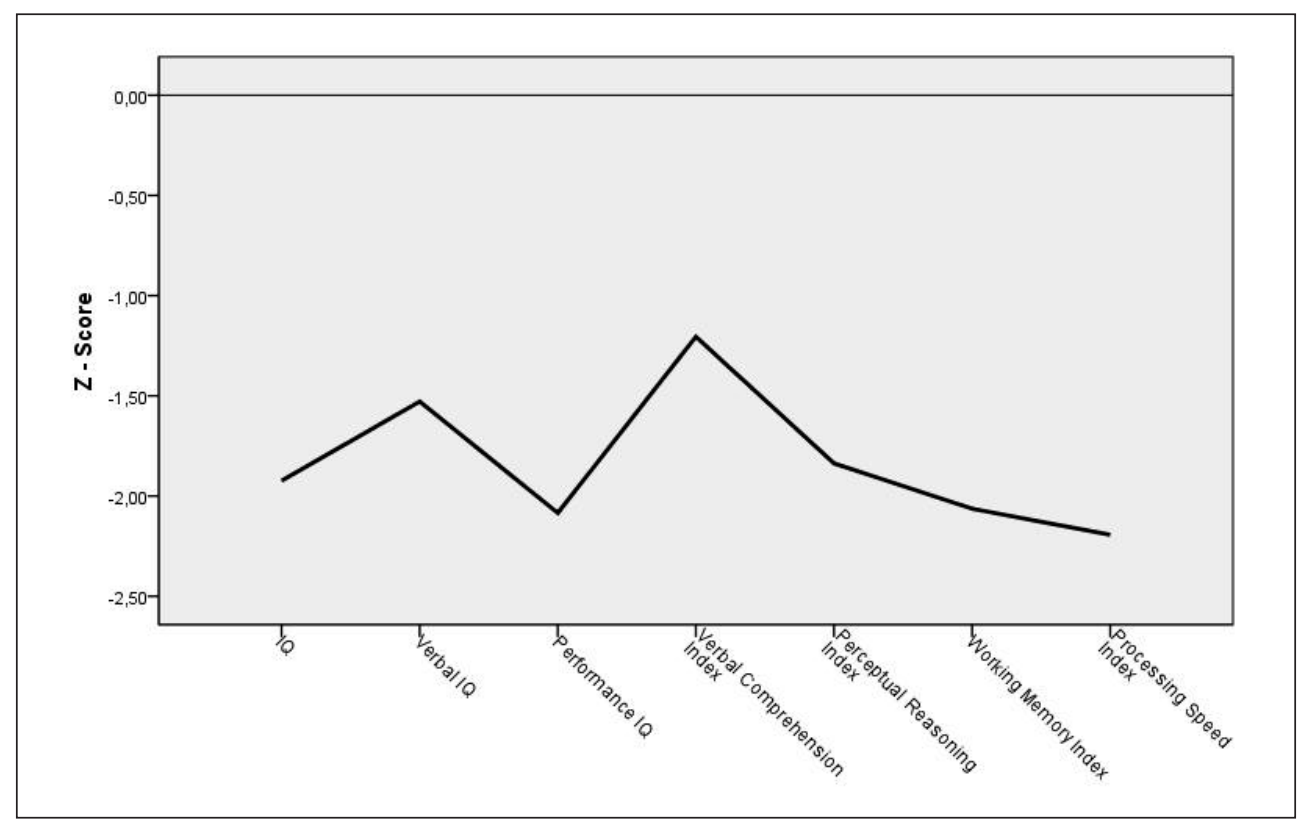

Figure 1. Mean Z - scores for the IQ of the WAIS-III in patients with chronic schizophrenia.

Table 3

Pairwise comparisons for the WAIS III-TR index.

\begin{tabular}{llcc} 
(I)Index & $(\mathrm{J})$ Index & Effect size & $P$ value \\
\hline Verbal Comprehension & Perceptual Organization & $8.56^{* *}$ & 0.002 \\
& Working Memory & $12.61^{* *}$ & 0.000 \\
& Processing Speed & $14.42^{* *}$ & 0.000 \\
\hline Perceptual Organization & Verbal Comprehension & $-8.56^{* *}$ & 0.002 \\
& Working Memory & $4.05^{*}$ & 0.013 \\
& Processing Speed & $5.85^{*}$ & 0.000 \\
\hline Working Memory & Verbal Comprehension & $-12.61^{* *}$ & 0.000 \\
& Perceptual Organization & $-4.05^{*}$ & 0.013 \\
& Processing Speed & 1.81 & 0.840 \\
\hline Processing Speed & Verbal Comprehension & $-14.42^{* *}$ & 0.000 \\
& Perceptual Organization & $-5.85^{* *}$ & 0.000 \\
& Working Memory & -1.81 & 0.840 \\
\hline
\end{tabular}

$* P<0.05, * * P<0.01$

${ }^{a}$ Bonferroni's adjustment. 
The Pearson correlation analysis showed that cognitive performance is strongly correlated with the Formal Thought Disorder. However, it is striking that this correlation was not established with the Verbal IQ and Verbal Comprehension IQ (Table 4). The severity of negative symptoms assessed globally was significantly correlated with a lower performance on the full intelligence scale, but specifically with the Performance IQ scores, Perceptual Organization and Working Memory. In particular, we observed a strong correlation between the severity of negative symptoms and poor performance in Processing Speed. Moreover, this study found that the severity of the alogia symptom, closely associated with language disorders, correlated strongly with all cognitive measures, including those within the Performance IQ. Avolition/apathy was strongly correlated with the results of the Performance IQ test, and the Perceptual Organization and Processing Speed index. No correlations of this type were observed in the present study between the symptoms of affective flattening, anhedonia-unsociability and attention and any of the cognitive measures (Table 5).

Table 4

Correlations between IQ Scores - Index Scores and positive psychotic symptoms in patients with chronic schizophrenia.

\begin{tabular}{lccccc}
$\begin{array}{l}\text { IQ Scores - Index Scores / } \\
\text { Psychotic Symptoms }\end{array}$ & SAPS & Hallucination & Delusions & $\begin{array}{c}\text { Bizarre } \\
\text { behavior }\end{array}$ & $\begin{array}{c}\text { Formal thought } \\
\text { disorder }\end{array}$ \\
\hline IQ Full Scale & -0.076 & -0.144 & 0.066 & -0.147 & $-0.348^{*}$ \\
Verbal IQ & -0.045 & -0.147 & 0.116 & -0.148 & -0.283 \\
Performance IQ & -0.150 & -0.143 & -0.069 & -0.159 & $-0.447 * *$ \\
Verbal Comprehension & -0.016 & -0.134 & 0.135 & -0.169 & -0.238 \\
Perceptual Organization & -0.178 & -0.202 & -0.142 & -0.178 & $-0.457 * *$ \\
Working Memory & -0.151 & -0.190 & -0.040 & -0.190 & $-0.431^{* *}$ \\
Processing Speed & -0.129 & -0.167 & -0.124 & -0.030 & $-0.482^{* *}$ \\
\hline
\end{tabular}

$* P<0.05, * * P<0.01$

Table 5

Correlations between IQ Scores - Index Scores and negative psychotic symptoms in patients with chronic schizophrenia.

\begin{tabular}{llclllc}
$\begin{array}{l}\text { IQ Scores - Index Scores / } \\
\text { Psychotic Symptoms }\end{array}$ & SANS & $\begin{array}{c}\text { Affective } \\
\text { flattening } \\
\text { or blunting }\end{array}$ & Alogia & $\begin{array}{c}\text { Avolition / } \\
\text { apathy }\end{array}$ & $\begin{array}{c}\text { Anhedonia / } \\
\text { asociality }\end{array}$ & Attention \\
\hline IQ Full Scale & $-0.340^{*}$ & -0.151 & $-0.507^{* *}$ & $-0.355^{*}$ & -0.078 & -0.176 \\
Verbal IQ & -0.293 & -0.147 & $-0.467^{* *}$ & -0.269 & -0.067 & -0.201 \\
Performance IQ & $-0.382^{*}$ & -0.134 & $-0.510^{* *}$ & $-0.478^{* *}$ & -0.084 & -0.118 \\
Verbal Comprehension & -0.250 & -0.183 & $-0.387^{*}$ & -0.124 & -0.066 & -0.203 \\
Perceptual Organization & $-0.344^{*}$ & -0.082 & $-0.477^{* *}$ & $-0.411^{* *}$ & -0.086 & -0.142 \\
Working Memory & $-0.366^{*}$ & -0.221 & $-0.492^{* *}$ & $-0.322^{*}$ & -0.041 & -0.249 \\
Processing Speed & $-0.419^{* *}$ & -0.258 & $-0.454^{* *}$ & $-0.498^{* *}$ & -0.111 & -0.026 \\
\hline
\end{tabular}

$* P<0.05,{ }^{*} * P<0.01$ 


\section{Discussion}

The aim of this study was to evaluate and describe the cognitive functioning of patients with chronic schizophrenia by administering the WAIS-III full scale, with the hypothesis that the performance of these patients would be significantly low compared to expectations. This hypothesis was tested and accepted, as we observed a significantly lower average yield in the different levels of the intelligence scale, with a qualitative assessment border. However, it is important to consider that, as stated in the WAIS-III manual, a low IQ may reflect a low level of intellectual functioning, but can also be determined by other factors such as linguistic or cultural discrepancy, the presence of distractibility or anxiety, low motivation, among others ${ }^{7}$.

In the case of patients with schizophrenia, there are many factors involved in cognitive performance and still no consensus regarding them, showing a great variability in the results of different studies. The highly variable results in some cases seem to be related to clinical and sociodemographic variables. Among these variables can be considered: the subtypes of schizophrenia, the current period of illness, years of illness and antipsychotic medication used. Many studies investigating the cognitive functioning with WAIS-III scale use outpatients stabilized in psychopathology ${ }^{16,17}$. However, in order to reduce the aforementioned variability we focused our research interest in patients with four different characteristics: they are inpatients, with presence of psychotic symptoms, suffering from chronic continuous manifestations of the disease, and on stable antipsychotic medication.

Coincidentally with the study reported in the technical manual for the WAIS-III, our results showed that higher scores were obtained in Verbal Comprehension and Perceptual Or- ganization, while the lowest scores were observed in the Working Memory and Processing Speed IQ, with the latter being the most affected $^{7}$. Multiple studies of specific cognitive domains in schizophrenia have shown that deficit in processing speed is the core element of cognitive impairment in schizophrenia ${ }^{1}$. However, it has been established that the strongest moderator of processing speed performance is observed between the daily dose of antipsychotic medication and the intensity of its effect on the encoding task, concluding that the antipsychotic dose has a significant effect on processing speed. Despite this assertion, even if the effects of medication were excluded, the deficit in processing speed would be significant ${ }^{1}$. In our study we did not analyze the relationship between the medication used and the performance in different cognitive scales, which is a limitation in our work; however, taking into account the previous results mentioned above, we consider it important to include this analysis in future.

One of the performance moderators considered in this study was the correlation analysis between WAIS-III cognitive measures and psychotic symptoms. The results showed that the overall severity of negative symptoms significantly affects cognitive functioning of chronic patients, but the slowing down of the cognitive processing was the most noticeably affected. The avolition/apathy symptomatic pair was strongly correlated with various cognitive measures, but the most significant correlation was observed with the results of the manipulative ability, and the perceptual organization and processing speed indices.

One of the most outstanding results is the strong negative correlation found between the severity of alogia and all measures of the WAIS-III, including tasks that are specifically manipulative, while the term alogia refers to the impoverishment of thought and 
cognition, and is inferred by the poverty of speech and poverty of language content ${ }^{14}$. These results are consistent with other results in this study that showed that the symptom of Formal Thought Disorder significantly correlated with almost all the WAIS-III measurements, but remarkably this symptom does not correlate with verbal measures. In this line of findings, Berenbaum ${ }^{17}$ found that both alogia and coherent speech impairment are associated with poor planning skills in cognitive tasks, which could explain the relationship between the severity of these symptoms and the poor performance in executive and manipulative tasks found in our study. Furthermore, we think that this strong association between the severity of alogia and general cognitive performance of patients can be explained from an integrated cognitive perspective, which is considered both a dysfunctional semantic system as severe problems in executive functioning of patients may underlie symptoms such as Formal Thought disorder and alterations in language fluency ${ }^{18}$.

Keefe ${ }^{19}$ has established that neuropsychological tests may provide, in addition to evidencing models of regional brain dysfunction in psychiatric disorders, an understanding of the relationship between central cognitive problems and symptoms, which can be used to identify cognitive predictors of the course of the disease and provide a method for discriminating between heterogeneous forms of certain psychiatric disorders. In this study we managed to describe the cognitive functioning of chronic patients using different indexes of the WAIS-III scale, a great benefit when trying to determine specific cognitive patterns.
Some of the limitations of this study relate to the type of analysis to be performed between the severity of symptoms and performance on cognitive tasks, while these results we cannot infer predictions regarding associations that may exist between these variables. However, we believe that our results provide useful information that can then be deepened. Moreover, we also believe that further studies are needed to compare cognitive performance and its association with psychotic symptoms, including chronic and acute patients.

\section{Acknowledgements}

We thank to Griselda Maidana, Florencia Di Yorio, Ana Molina, Cristina Moreno, Sabrina Trovatto and Fernando Gay, for their generous help while we testing the patients.

\section{Conflict of interest}

The authors declare that they have no competing interests.

\section{Role of the funding source}

FSG was supported by the Doctoral Scholarship Type II 2013 of the Secretary of Science and Technology (SECyT), Universidad Nacional de Córdoba (UNC). SECyT and UNC have not participated in study design, collection, analysis and interpretation of data, writing of the report, or the decision to submit the paper for publication. 


\section{References}

1. Knowles E, David AS, Reichenberg A. Processing Speed Deficits in Schizophrenia: Reexamining the Evidence. Am J Psychiatry. 2010; 167 (7): 828-835.

2. Kehagia AA, Murray GK, Robbins TW. Learning and cognitive flexibility: Frontostriatal function and monoaminergic modulation. Current Opinion in Neurobiology. 2010; 20 (2): 199-204.

3. Sepede G, Ferretti A, Gianni Perrucci M, Gambi F, Di Donato F, Nuccetelli F, et al. Altered brain response without behavioral attention deficits in healthy siblings of schizophrenic patients: An event-related fMRI study. NeuroImage. 2010; 49 (1): 1080-1090.

4. Kuperberg GR, Heckers S. Schizophrenia and cognitive function. Current Opinion in Neurobiology. 2000; 10: 205-210.

5. Buchanan RW, Davis M, Goff D, Green MF, Keefe RS, Leon AC, et al. A summary of the FDA-NIMH-MATRICS workshop on clinical trial design for neurocognitive drugs for schizophrenia. Schizophr Bulletin. 2005; 31 (1): 5-19.

6. Keefe RS. Should cognitive impairment be included in the diagnostic criteria for schizophrenia? World Psychiatry. 2008; 7: 22-28.

7. Wechsler D. Manual de Administración y Puntuación. Test de Inteligencia para Adultos WAIS-III. Tercera edición. Buenos Aires: Paidós. 2006.

8. Ryan JJ, Weilage ME, Spaulding WD. Accuracy of the seven subtest WAIS-R short form in chronic schizophrenia. Schizophrenia Research. 1999; 39 (1): 79-83.

9. Kurtz MM, Donato J, Rose J. Crystallized verbal skills in schizophrenia: Relationship to neurocognition, symptoms, and functional status. Neuropsychology. 2011; 25 (6): 784-791.

10. Dickinson D, Ramsey ME, Gold JM. Overlooking the obvious: A meta-analytic comparison of digit symbol coding tasks and other cognitive measures in schizophrenia. Arch Gen Psychiatry. 2007; 64: 532-542.

11. Henry JD, Crawford JR. A meta-analytic review of verbal fluency deficits in schizophrenia relative to other neurocognitive deficits. Cogn Neuropsychiatry. 2005; 10: 1-33.
12. Hughes C, Kumari V, Soni W, Das M, Binnerman B, Drozd S, et al. Longitudinal study of symptoms and cognitive function in chronic schizophrenia. Schizophr Res. 2002; 59: $137-146$

13. American Psychiatric Association. Diagnostic and Statistical Manual of Mental Disorders, Fourth Edition: DSM-IV-TR. 2000.

14. Andreasen NC. The Scale for the Assessment of Negative Symptoms (SANS). Iowa City, Iowa: The University of Iowa. 1984.

15. Andreasen NC. The Scale for the Assessment of Positive Symptoms (SAPS). Iowa City, IA: The University of Iowa. 1984.

16. Blyler CR, Gol JM, Iannone VN, Buchanan RW. Short form of the WAIS-III for use with patients with schizophrenia. Schizophrenia Research. 2000; 46 (2): 209-215.

17. Berenbaum H, Kerns JG, Vernon LL, Gomez JJ. Cognitive correlates of schizophrenia signs and symptoms: III. Hallucinations and delusions. Psychiatry Research. 2008; 159 (1-2): 163-166.

18. Holshausen K, Harvey PD, Elvevåg B, Foltz PW, Bowie CR. Latent semantic variables are associated with formal thought disorder and adaptive behavior in older inpatients with schizophrenia. Cortex. 2014; 55: 88-96.

19. Keeffe RS. The contribution if neuropsychology to psychiatry. The American Journal of Psychiatry. 1995; 152: 6-15.

Corresponding author:

Flavia S. Galaverna

Laboratory of Experimental Psychology

Faculty of Psychology

National University of Córdoba

Enfermera Gordillo esquina Enrique Barros

Ciudad Universitaria, CP X5000GYA

Córdoba

Argentina

Tel.:+ 543514344984

Fax: + 543514334064

E-mail: fgalaverna@psyche.unc.edu.ar 